\title{
Colloidal Phase-Change Materials: Synthesis of Monodisperse GeTe Nanoparticles and Quantification of Their Size-Dependent Crystallization
}

\author{
Olesya Yarema, ${ }^{\dagger}$ Aleksandr Perevedentsev, ${ }^{\ddagger}$ Vladimir Ovuka, ${ }^{\dagger}$ Paul Baade, ${ }^{\dagger}$ Sebastian Volk, ${ }^{\dagger}$ \\ Vanessa Wood, ${ }^{* \dagger}$ and Maksym Yarema ${ }^{*}, \dagger$ \\ ${ }^{\dagger}$ Materials and Device Engineering Group, Department of Information Technology and Electrical Engineering, ETH Zurich, \\ Gloriastrasse 35, CH-8092 Zurich, Switzerland \\ ${ }^{\ddagger}$ Polymer Technology, Department of Materials, ETH Zurich, Vladimir-Prelog-Weg 5, CH-8093 Zurich, Switzerland
}

Supporting Information

\begin{abstract}
Phase-change memory materials refer to a class of materials that can exist in amorphous and crystalline phases with distinctly different electrical or optical properties, as well as exhibit outstanding crystallization kinetics and optimal phase transition temperatures. This paper focuses on the potential of colloids as phase-change memory materials. We report a novel synthesis for amorphous GeTe nanoparticles based on an amide-promoted approach that enables accurate size control of GeTe nanoparticles between 4 and $9 \mathrm{~nm}$, narrow size distributions down to $9-10 \%$, and synthesis upscaling to reach multigram chemical yields per batch. We then quantify the crystallization phase transition for GeTe nanoparticles, employing high-temperature X-ray diffraction, differential

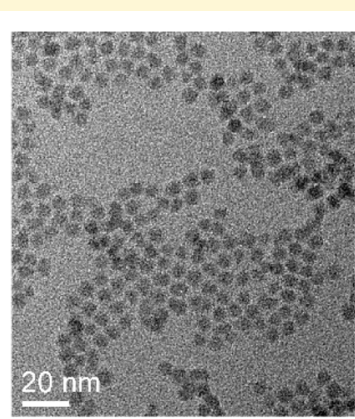

Colloidal GeTe nanoparticles

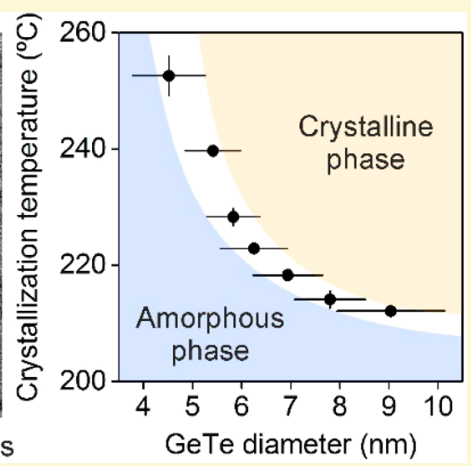
scanning calorimetry, and transmission electron microscopy. We show that GeTe nanoparticles crystallize at higher temperatures than the bulk GeTe material and that crystallization temperature increases with decreasing size. We can explain this size-dependence using the entropy of crystallization model and classical nucleation theory. The size-dependences quantified here highlight possible benefits of nanoparticles for phase-change memory applications.
\end{abstract}

\section{INTRODUCTION}

Phase-change memory is a transistor-free data storage technology that leverages crystallization and melting phase transitions, using the resistivity contrast between the amorphous and crystalline phases of the material as the digital 0 and 1.1,2 A miniaturization of phase-change memory chips will ultimately bring the size of memory cells to sub-10 nm regime, where the phase transition temperatures become a function of size. ${ }^{3}$ Colloidal nanoparticles are a convenient material system to study size-dependent phase transitions ${ }^{4,5}$ due to their fast synthesis, providing monodisperse templatefree nanoparticles of a specific size. ${ }^{6}$ Furthermore, colloidal nanoparticles may themselves be used as the building blocks for future phase change memory cells.

This paper focuses on Germanium(II) telluride, GeTe, a prototype phase-change material, which is well studied in bulk and thin films. GeTe offers distinct crystallization and melting temperatures $\left(T_{\text {cryst,bulk }}=170{ }^{\circ} \mathrm{C} ; T_{\text {melt,bulk }}=725{ }^{\circ} \mathrm{C}\right),{ }^{3,7}$ pronounced resistivity contrast between amorphous and crystalline phases $\left(R_{\text {amorph }} / R_{\text {cryst }}>1000\right){ }^{8}$ and suitable crystallization kinetics (i.e., fast at high temperatures and slow at room temperature, $k^{350 \circ \mathrm{C}} \approx 1 \mathrm{~m} / \mathrm{s}, k^{30 \circ \mathrm{C}} \ll 1 \mathrm{~nm} /$ year). ${ }^{9} \mathrm{GeTe}$ and its ternary alloys are also of interest for other applications, such as ferroelectric and multiferroic, thermoelectric, and infrared semiconductor applications. ${ }^{10,11}$

Synthesis of colloidal GeTe nanoparticles is not well developed. Depending on the reaction conditions, GeTe nanoparticles can exhibit either an amorphous or crystalline structure upon synthesis. Amorphous GeTe nanoparticles were reported by Caldwell et al. (sizes from 1.8 to $3.4 \mathrm{~nm}$ in diameter) ${ }^{12}$ and by Arachchige et al. (sizes $\geq 8.7 \mathrm{~nm}$ in diameter). ${ }^{13}$ Crystalline $\mathrm{GeTe}$ nanoparticles are generally larger in size, and have been reported as 8 and $17 \mathrm{~nm}$ dots, ${ }^{14} 100 \mathrm{~nm}$ octahedrons, or $350 \mathrm{~nm}$ cubes. ${ }^{15,16}$ Since we aim to study the effect of nanoparticle size on the crystallization phase transition, here we wish to obtain amorphous GeTe nanoparticles.

Crystallization temperature of $\mathrm{GeTe}$ nanoparticles is known to deviate from the bulk value of $170{ }^{\circ} \mathrm{C}$, however, the size dependent crystallization behavior of colloidal nanoparticles has never been quantitatively explained. Caldwell et al. ${ }^{12}$ and Arachchige et al. ${ }^{13}$ observed a general trend-crystallization

Received: June 26, 2018

Revised: August 19, 2018

Published: August 20, 2018 
temperature gradually increases for smaller GeTe nanoparticles, reaching up to $400{ }^{\circ} \mathrm{C}$ for $1.8 \mathrm{~nm}$ GeTe clusters. Part of the challenge in explaining the size-dependence of the crystallization temperature comes from the fact that it is opposite to the trend in melting point, which decreases with decreasing nanoparticle size and which is explained by a higher energy per atom due to the increasing number of surface atoms as nanoparticle size decreases. ${ }^{17,18}$ Furthermore, coalescence of nanoparticles occurs near the crystallization temperatures, and most experimentation methodologies do not separate crystallization and coalescence phenomena. ${ }^{12,13}$

In this paper, we report a new one-step synthesis of amorphous GeTe nanoparticles with accurate size control between 4 and $9 \mathrm{~nm}$, which closes the size gap between previous reports. ${ }^{12,13} \mathrm{We}$ then use these nanoparticles to perform the first quantitative study of the size-dependent crystallization of template-free $\mathrm{GeTe}$ nanoparticles. To separate coalescence and crystallization, we use several complementary methods, such as constant heating ramp Xray diffraction, differential scanning calorimetry, and ex-situ heating transmission electron microscopy. We then explain the experimentally observed size-dependence of GeTe crystallization with thermodynamic model and classical nucleation theory. Finally, with our results, we calculate the temperature window, reduced crystallization temperature, and the power consumption to evaluate how size effects can be used to tune the properties and performance of phase-change memory devices.

\section{EXPERIMENTAL SECTION}

Materials. $\mathrm{GeI}_{2}(99.99 \%)$ and $\mathrm{Na}_{2} \mathrm{Te}(99.9 \%)$ were purchased from $\mathrm{ABCR}$, tri- $n$-octylphosphine (TOP, 97\%), Te (broken ingots, $99.999 \%)$, and $\mathrm{K}_{2} \mathrm{~S}$ (95\%) from STREM, oleic acid (90\%), chloroform (99\%), ethanol (99.8\%), n-butylamine (99.5\%), $N, N$ dimethylformamide (DMF, 99.8\%), hexane (95\%), toluene (99.8\%), and $\mathrm{LiI}$ (99.9\%) from Sigma-Aldrich, $\mathrm{LiN}\left(\mathrm{SiMe}_{3}\right)_{2}$ (95\%) from Acros Organics. Oleic acid was dried at $100{ }^{\circ} \mathrm{C}$ for $1 \mathrm{~h}$ from water residues and all other chemicals were of anhydrous grade and were used asreceived.

Synthesis of GeTe Nanoparticles. In a typical synthesis of $7 \mathrm{~nm}$ GeTe nanoparticles, anhydrous $\mathrm{GeI}_{2}(107 \mathrm{mg}, 0.33 \mathrm{mmol}$ ) was dissolved in tri- $n$-octylphosphine (TOP, $7.5 \mathrm{~mL}$ ) in the glovebox and transferred to the prepumped reaction flask, connected to standard vacuum manifold. This mixture was additionally purified under vacuum at $100{ }^{\circ} \mathrm{C}$ for $30 \mathrm{~min}$, after which it was filled with $\mathrm{N}_{2}$ and heated up to injection temperature of $280{ }^{\circ} \mathrm{C}$. Meanwhile, an injection mixture was prepared by mixing two TOP-based stock solutions $-0.8 \mathrm{~mL}$ of $1 \mathrm{M}$ TOP:Te $(0.8 \mathrm{mmol}$ of Te, in total $)$ and 0.5 $\mathrm{mL}$ of $1.6 \mathrm{M} \mathrm{LiN}\left(\mathrm{SiMe}_{3}\right)_{2}(0.8 \mathrm{mmol}$ of amide, in total $)$ - and swiftly added to the reaction mixture as soon as its temperature reached injection set value. After injection, the temperature of the reaction mixture drops by about $20^{\circ} \mathrm{C}$ and was kept as such for another $1-2$ min. The solution changes its color to deep brown during this time and reaction was terminated by rapidly cooling the flask with pressurized air and, later, with cold water bath. Once cooled to room temperature, the crude solution of GeTe nanoparticles was transferred air-free to the glovebox, where anhydrous chloroform $(10 \mathrm{~mL})$ and dried oleic acid $(1 \mathrm{~mL})$ were added to it. The mixture was shaken rigorously and set aside for 5-10 min, allowing for the completion of oleate shell formation. The oleate-covered GeTe nanoparticles were precipitated with anhydrous ethanol $(30 \mathrm{~mL})$ and separated by centrifugation at $6000 \mathrm{~g}$ for $5 \mathrm{~min}$. The obtained pellet of GeTe nanoparticles was dispersed in anhydrous chloroform, forming a longterm stable colloidal solution.

The size of GeTe nanoparticles can be controlled by the amount of amide salt and by the injection temperature, whereas the selection of growth time between 0.5 and $5 \mathrm{~min}$ has a relatively minor effect. The $50 \times$ upscaled synthesis of GeTe nanoparticles follows the same procedures as explained above, while using underpressure-governed hot-injection technique for fast addition of injection mixture. ${ }^{19}$

Ligand Exchange Process or GeTe Nanoparticles. In analogy to previously published protocols, several inorganic salts ( $\mathrm{LiI}, \mathrm{GeI}_{2}$, $\mathrm{K}_{2} \mathrm{~S}$, or $\mathrm{Na}_{2} \mathrm{Te}$ ) were used to remove initial organic ligands (oleic acid) from the surface of the GeTe nanoparticles. ${ }^{20,21}$ In a typical ligand-exchange process using $\mathrm{GeI}_{2}$, a starting solution of $\mathrm{GeTe}$ nanoparticles $(1 \mathrm{~mL}$, approximately $6 \mathrm{mg} / \mathrm{mL})$, was precipitated with ethanol $(3 \mathrm{~mL})$, centrifuged at $9000 \mathrm{~g}$ for $3 \mathrm{~min}$, and dispersed in toluene $(200 \mu \mathrm{L})$. This solution was mixed with $\mathrm{GeI}_{2} / \mathrm{DMF}$ solution $(3 \mathrm{~mL}, 20 \mathrm{mg} / \mathrm{mL}$ ), and $15 \mathrm{~mL}$ of hexane, forming a 2-phase solvent system. The solvent mixture was rigorously shaken for few minutes, during which GeTe nanoparticles transfer to DMF phase. The hexane phase was then decanted and the GeTe/DMF solution was purified 4 more times with hexane to ensure complete removal of oleic acid. In addition, after the second washing step, $3 \mathrm{~mL}$ of DMF was added, to ensure that nanoparticles do not cluster. Afterward, $\mathrm{GeI}_{2}$-covered $\mathrm{GeTe}$ nanoparticles were precipitated with chloroform (1:3 v:v), centrifuged at $9000 \mathrm{~g}$ for $3 \mathrm{~min}$, and redissolved in $n$-butylamine $(0.2$ $\mathrm{mL}$ ).

High-temperature X-ray diffraction (XRD). GeTe nanoparticles were precipitated with ethanol and centrifuged. GeTe pellets were mixed with a heat-stable polymer to spatially separate GeTe nanoparticles, and loaded onto an inconel holder. High-temperature XRD was performed on Rigaku SmartLab 9 kW System, equipped with rotating $\mathrm{Cu}$ anode and 2D solid state detector (HyPix-3000 SL). High-temperature stage (Anton Paar) consists of a ceramic plate, which is shielded with a carbon dome. The high-temperature XRD measurements were performed under constant heating ramp and with the temperature precision of $\pm 1{ }^{\circ} \mathrm{C}$. All nanoparticle handling and measurements were carried out under $\mathrm{N}_{2}$ atmosphere.

Differential Scanning Calorimetry (DSC). Thermal analysis by DSC was carried out using a Mettler Toledo DSC822e instrument calibrated against indium standards. All measurements were performed under $\mathrm{N}_{2}$ blanket. Freshly prepared GeTe nanoparticle dispersions in chloroform were added into aluminum crucibles and immediately heated to $90{ }^{\circ} \mathrm{C}$ for $5 \mathrm{~min}$ to evaporate the residual solvent, following which the samples were thermally cycled in the -40 to $350{ }^{\circ} \mathrm{C}$ range at different heating rates.

Electron Microscopy. Energy dispersive X-ray (EDX) spectroscopy was performed on an FEI Quanta 200 SEM microscope (30 kV). Transmission electron microscopy (TEM) images were taken by an FEI Tecnai F30 transmission electron microscope $(300 \mathrm{kV})$. Ex situ heating TEM analysis was carried out on a series of TEM grids, each heated to a specific temperature for $1 \mathrm{~min}$ under $\mathrm{N}_{2}$ atm.

Fourier-Transform Infrared (FTIR) Spectroscopy. GeTe nanoparticles were drop-cast on $\mathrm{ZnSe}$ transparent windows and measurements were performed on a Bruker V70 system with an InGaAs detector.

Electrical Characterization. $\mathrm{GeI}_{2}$-covered GeTe nanoparticles in $n$-butylamine were filtered through $0.2 \mu \mathrm{m}$ PTFE filter and spincoated on interdigitated Au electrode structures. Each interdigitated electrode pair consists of 10 electrode fingers, approximately $2.1 \mathrm{~mm}$ long, $57 \mu \mathrm{m}$ wide, and spaced $36 \mu \mathrm{m}$ apart. Electrode characterization was done using a Keithley 2400 SMU, with voltage double sweeps spanning from -1 to $1 \mathrm{~V}$ in 81 steps, with $100 \mathrm{~mA}$ current compliance. Electrical characterization of GeTe nanoparticle thin films was done in an inert atmosphere $\left(\mathrm{N}_{2}\right.$-filled glovebox $)$.

\section{RESULTS}

Synthesis. To prepare monodisperse GeTe nanoparticles, we employ an amide-promoted synthetic approach, which was previously applied to binary and ternary chalcogenide nanocrystals. ${ }^{22-24}$ An amide salt, injected to the reaction mixture along with the chalcogen precursor, increases the nucleation rate and thus yields smaller sizes of nanoparticles, if compared to analogous reaction conditions without an 


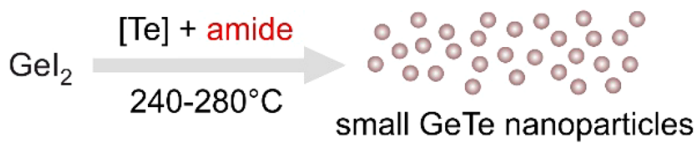

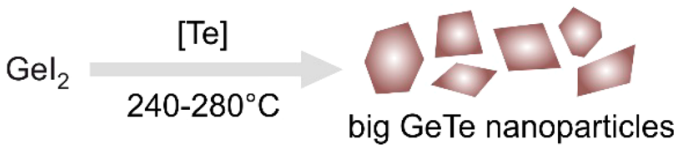

C

[amide] $<$ [iodide]

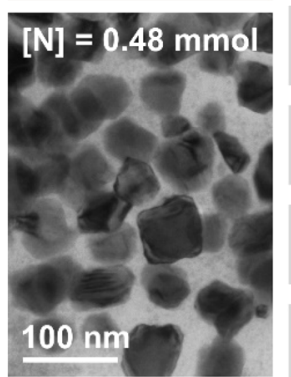

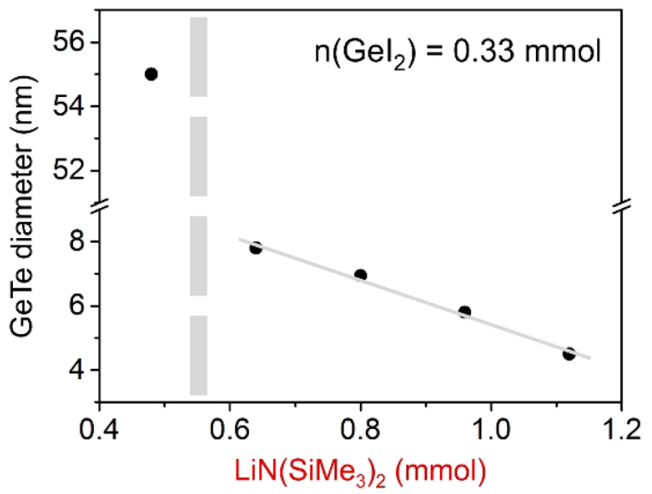

[amide] $\geq$ [iodide]

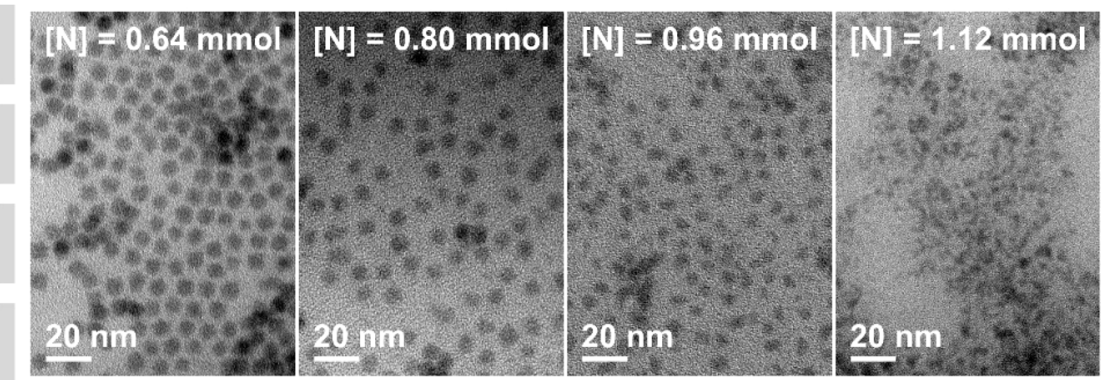

Figure 1. (a) Schematic illustration of amide-promoted synthesis and direct precursor reaction for GeTe nanoparticles. (b) Size control is achieved by tuning the amount of amide salt, $\mathrm{LiN}\left(\mathrm{SiMe}_{3}\right)_{2}$. (c) TEM images show this size dependence.

addition of amide promoter (Figure 1a). This method is especially useful for telluride nanoparticles because it can effectively separate nucleation and growth of nanoparticles, which is challenging when highly reactive tellurium precursors are employed. ${ }^{25}$ Importantly, the amount of amide can be used as a handle for size control-an increase of amide concentration leads to higher nucleation rates and hence smaller sizes of nanoparticles. ${ }^{22-24}$

By tuning the amount of coinjected amide salt, we achieve GeTe nanoparticles sizes between 4 and $8 \mathrm{~nm}$ (Figure $1 \mathrm{~b}$ ). In accordance with previous reports, the amide concentration has to be higher than that of the initial iodide anions, enabling full conversion to the germanium-amide intermediate followed by its fast reaction with the chalcogen (tellurium) precursor. ${ }^{24,25}$ Small amounts of $\mathrm{LiN}\left(\mathrm{SiMe}_{3}\right)_{2}$ result in slower nucleation on fewer nucleation centers, leaving sufficient amounts of starting precursors for direct reaction and yielding broadly dispersed large-size GeTe nanoparticles. Figure 1c illustrates these findings, showing a series of TEM images of GeTe products, prepared with different amounts of $\mathrm{LiN}\left(\mathrm{SiMe}_{3}\right)_{2}$ and otherwise identical reaction conditions. In particular, amide-promoted synthesis yields monodisperse GeTe nanoparticles with size distributions as narrow as 9\% (Figure S1 of the Supporting Information, SI). Additional size tunability can be attained by regulating the injection temperature. The temperature dependence follows classical kinetics-lower injection (and growth) temperatures result in slower growth and thus smaller GeTe nanoparticles (Figure S2). Changing the growth time has only a minor effect on the size and size distribution of GeTe nanoparticles (Figure S3), which suggests fast completion of the reaction and high chemical yields of the process. The synthesis of GeTe nanoparticles is described in the Experimental Section in more detail.
As-synthesized small GeTe nanoparticles exhibit an amorphous (glass-like) structure, which is indicated by absence of diffraction contrast on TEM images (Figures S2 and S3) and, as shown later, by X-ray diffraction spectra and highresolution TEM images. From EDX we find that GeTe nanoparticles are close to stoichiometric, with $1: 1 \mathrm{Ge}: \mathrm{Te}$ atomic ratio, suggesting that the reaction system is well balanced and prevents oxidation of $\mathrm{Ge}^{2+}$ ions to its more stable $\mathrm{Ge}(+4)$ oxidation state. A typical EDX spectrum of GeTe nanoparticles is shown in Figure S4.

To robustly characterize a material and consider for further applications, it is important to be able to prepare it in large quantities with high chemical yield. ${ }^{26}$ To address this challenge, we employed underpressure-governed hot-injection technique that we previously developed. ${ }^{19}$ This method allows for fast injection from the addition funnel, run by applying mild vacuum to the reaction flask (TEM analysis and upscaling setup is shown in Figure 2). Using underpressure-governed injection, we successfully upscale the GeTe recipe by a factor of 50 and achieve $2 \mathrm{~g}$ of nanoparticles per batch, while maintaining the narrow size distribution of GeTe nanoparticles. For the same precursor ratios, the size of GeTe nanoparticles is slightly smaller than that obtained by smallscale synthesis (Figure S5). This can be explained by larger temperature drop after large-scale injection, associated with faster injection rates and boiling of the reaction mixture under mild vacuum. ${ }^{19}$

Measuring Crystallization Temperature. To demonstrate how we quantify the crystallization temperature, we work with the $6 \mathrm{~nm}$ sized GeTe particles produced from the upscaled synthesis. To estimate the crystallization point, we perform high-temperature X-ray diffraction measurements (HT-XRD), heating GeTe nanoparticles under $\mathrm{N}_{2}$ atmosphere at a constant ramp of $7^{\circ} \mathrm{C} / \mathrm{min}$ (Figure S6). The results of 


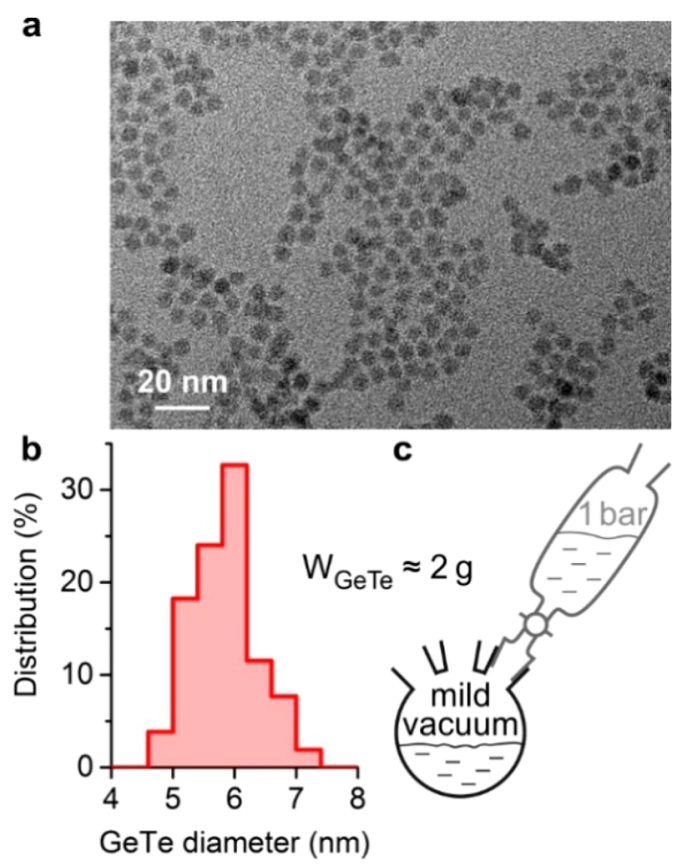

Figure 2. (a) TEM image, (b) size distribution, (c) and setup of large-scale synthesis of $6 \mathrm{~nm} \mathrm{GeTe}$ nanoparticles. The total yield of reaction, $W_{\mathrm{GeTe}}$, is about $2 \mathrm{~g}$.

HT-XRD are summarized in Figure 3. As-synthesized GeTe nanoparticles retain their amorphous structure up to temperatures $>200{ }^{\circ} \mathrm{C}$, as seen by the absence of Bragg reflections on $\mathrm{XRD}$ spectra. Upon further heating, X-ray peaks appear and match the rhombohedral modification of bulk GeTe well (Figures 3a and S7). ${ }^{15}$ The peak corresponding to the main Bragg reflection (202) is shown in Figure $3 b$, and its intensity is plotted as a function of sample temperature (Figure $3 \mathrm{c}$ ). This curve has a sigmoidal growth shape with its two plateau regions corresponding to amorphous and crystalline structures of GeTe nanoparticles (Figure 3d). The shaded region in Figure $3 \mathrm{c}$ represents the temperature range where crystallization of $\mathrm{GeTe}$ nanoparticles occurs. By analyzing the width of the (202) reflection, we find that crystalline domain size increases up to $25 \mathrm{~nm}$ in this region (Figure S8), which indicates that coalescence (i.e., sintering) of neighboring $\mathrm{GeTe}$ nanoparticles also occurs in the same temperature range as crystallization.

To distinguish between coalescence and crystallization and to gain a quantify understanding of crystallization, we perform differential scanning calorimetry (DSC) measurements on 6 $\mathrm{nm}$ GeTe nanoparticles, heated under $\mathrm{N}_{2}$ atmosphere at different rates (Figure 4a). We observe an exothermal signal, comprising two closely spaced peaks. Figure $4 \mathrm{~b}$ plots these peak positions (extracted by taking the second derivatives of the thermograms) as a function of heating rate. On the basis of ex situ heating TEM experiments (Figure S9), we assign the lower temperature process to crystallization and the higher temperature to coalescence. Kissinger analysis can be applied to extract activation energies of the processes (Figure 4c). Crystallization has a higher activation energy $(3.29 \mathrm{eV})$ than coalescence $(2.79 \mathrm{eV})$, which is in agreement with the literature for $\mathrm{Ca}-\mathrm{Mg}$ alumosilicates and telluride glasses. ${ }^{27,28}$

We hypothesize that the slightly higher coalescence temperature is due to the presence of an organic shell around the nanoparticles. The shell spaces nanoparticles (by approximately $1 \mathrm{~nm}$, Figures $1 \mathrm{c}$ and $2 \mathrm{a}$ ), which comprises a physical gap for atomic diffusion and coalescence. This assumption is supported by the fact that the relative magnitude (i.e., enthalpy) of crystallization and coalescence peaks in the DSC thermograms is dependent on the heating rate: faster heating increases the contribution of the crystallization exotherm because there is less time for sintering (Figure 4a).

To confirm that the organic shell enables us to observe isolate the temperature-dependent crystallization process, we show that we can instead cause coalescence followed by
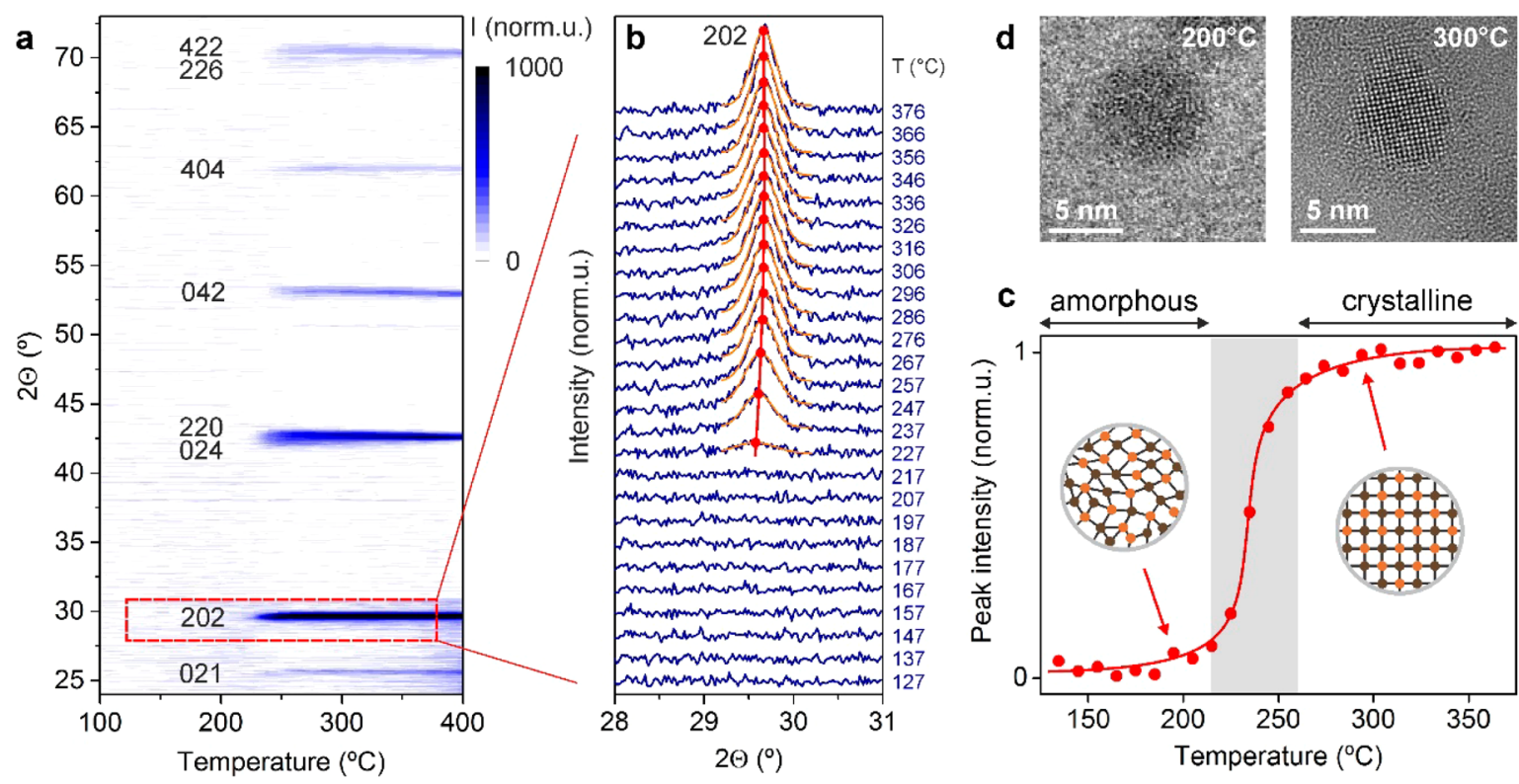

Figure 3. (a) High-temperature X-ray diffraction map for $6 \mathrm{~nm} \mathrm{GeTe}$ nanoparticles heated at a constant rate of $7{ }^{\circ} \mathrm{C} / \mathrm{min}$. (b) Zoom-in of panel (a) showing the (202) Bragg reflection indicative of rhombohedral GeTe. (c) Intensity of the (202) peak as a function of temperature, extracted from Gaussian fits of (b). (d) High-resolution transmission electron microscopy images of GeTe nanoparticles heated to $200{ }^{\circ} \mathrm{C}$ and $300{ }^{\circ} \mathrm{C}$ for 1 min. 
a
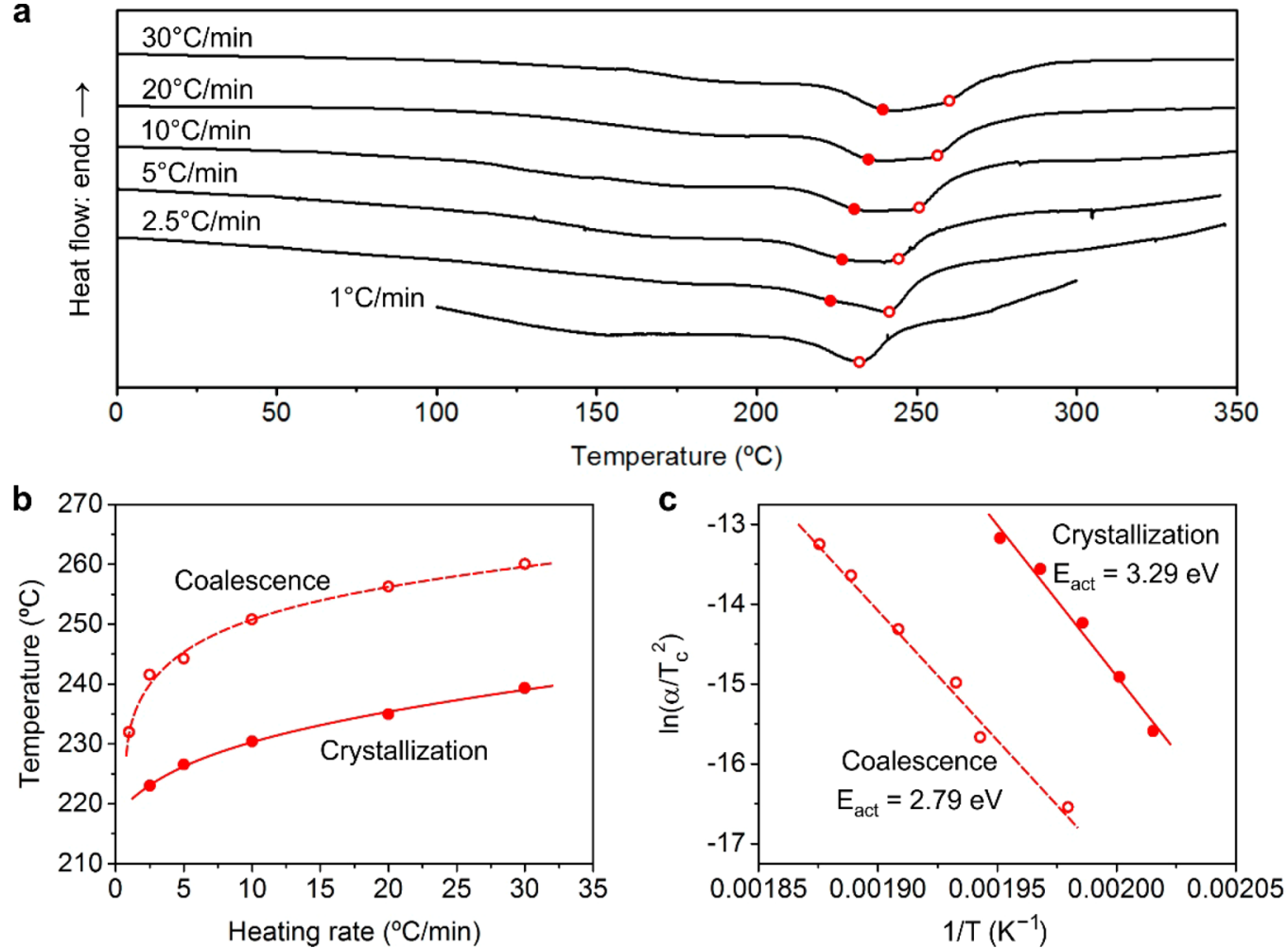

Figure 4. (a) First-heating differential scanning calorimetry thermograms recorded for $6 \mathrm{~nm}$ GeTe nanoparticles at the indicated rates. The main exotherms $\left(210-270{ }^{\circ} \mathrm{C}\right.$ range) show contributions from crystallization and coalescence with the corresponding peak positions indicated with solid and open circles, respectively. (b) Peak temperatures for crystallization and coalescence of GeTe nanoparticles as a function of heating rate. (c) Kissinger plot for the peak temperatures of crystallization and coalescence transitions. Activation energies are extracted from the linear fits in (c).

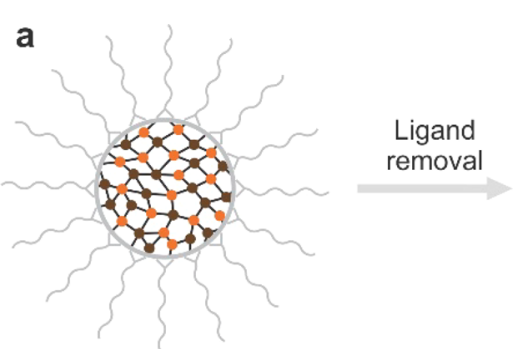

b
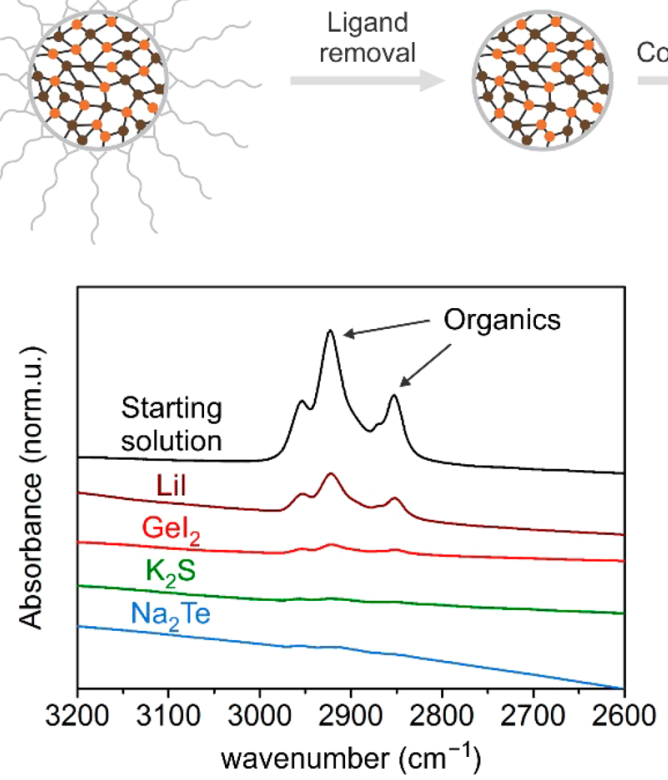

Coalescence
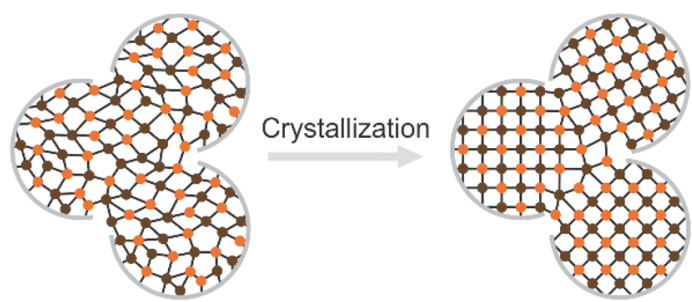

C

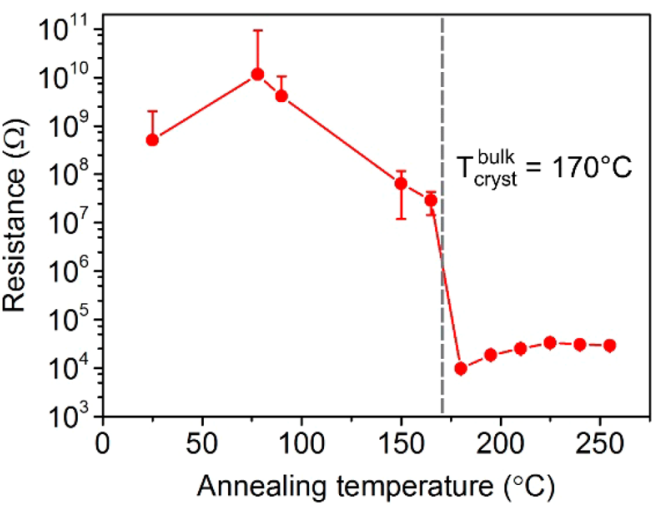

Figure 5. (a) Schematic representation of coalescence and crystallization of GeTe nanoparticles after removal of protective organic ligands. (b) Fourier-transform infrared spectra of GeTe nanoparticles before (starting solution) and after ligand exchange with the indicated inorganic salts. (c) Resistance of a $\mathrm{GeI}_{2}$-covered GeTe nanoparticle thin film annealed at different temperatures. Since coalescence happens before crystallization in these inorganic anion treated materials (see panel (a)), a resistance drop of $>1000$ is observed around the crystallization point of bulk GeTe.

crystallization by replacing the steric organic ligands with short inorganic anions (Figure 5a). Fourier-transform infrared spectroscopy confirms nearly complete elimination of organic shell for several different ligand-exchange protocols (Figure 5b). ${ }^{20,21}$ We perform temperature-dependent resistance measurements of $\mathrm{GeI}_{2}$-covered $\mathrm{GeTe}$ nanoparticle thin films 


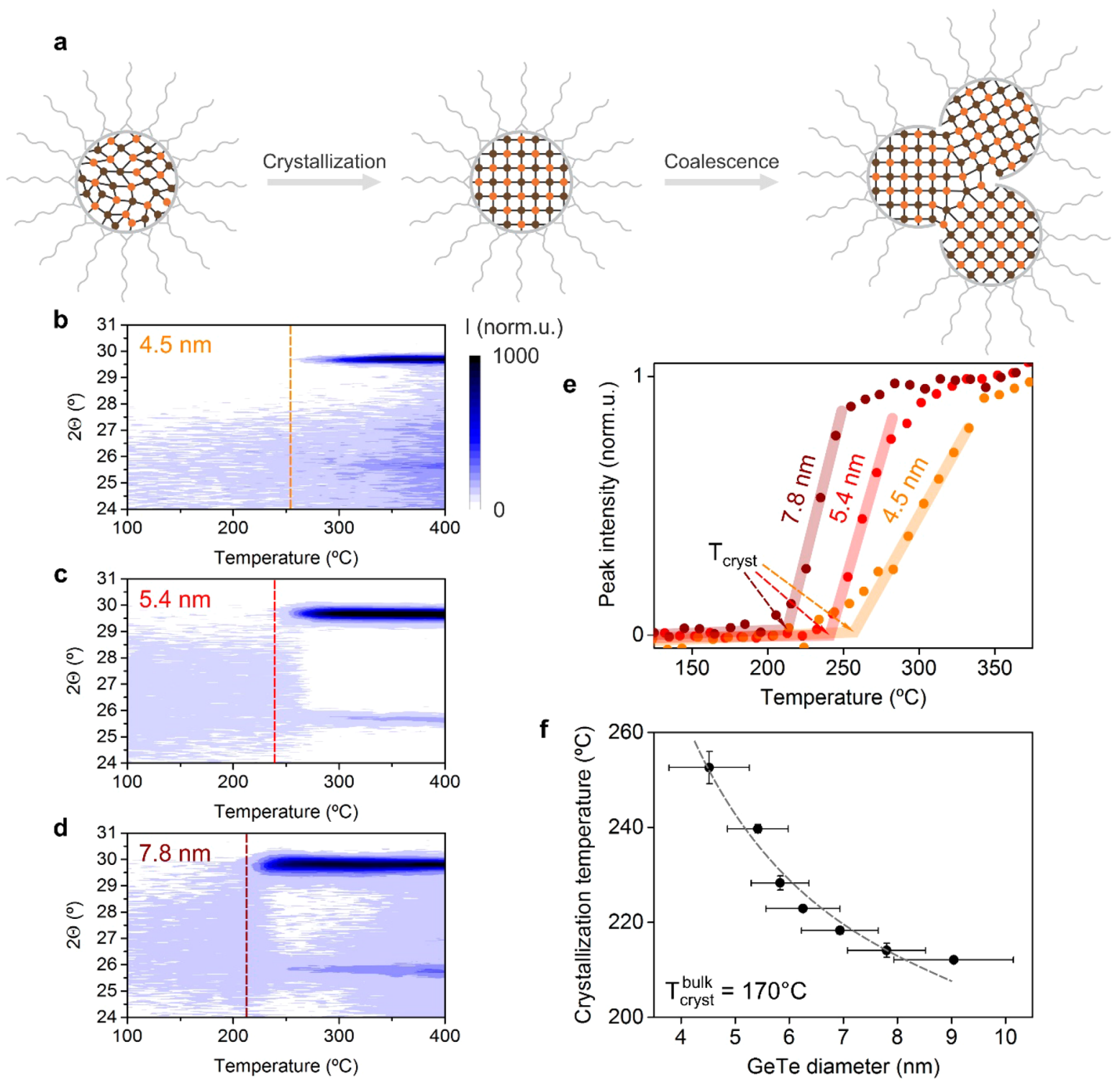

Figure 6. (a) Schematic representation of crystallization and coalescence of GeTe nanoparticles, when covered with organic ligands. (b-d) Hightemperature X-ray diffraction maps and (e) extracted intensity profiles of (202) Bragg reflection for GeTe nanoparticles with the indicated average sizes. (f) Size dependence of crystallization temperature for GeTe nanoparticles extracted from peak intensity profiles in (e) and fitted with entropy of crystallization model.

(Figure 5c) and observe a pronounced resistance drop at the bulk crystallization temperature of $\mathrm{GeTe}$ of $170{ }^{\circ} \mathrm{C}$. This indicates that coalescence of the film took place prior to or at the crystallization temperature, and that following coalescence the GeTe film behaves as a bulk material (Figure 5c).

\section{DISCUSSION}

Size-Dependent Crystallization Temperature. In the section above, we explain how we determine the crystallization temperature of GeTe nanoparticles using high-temperature XRD, DSC, and ex situ TEM (Figures 3, 4, and S6-S9). In particular, we find that the two processes-crystallization and coalescence of GeTe nanoparticles-happen almost simultaneously. When heating up the nanoparticles with an organic shell, crystallization of GeTe nanoparticles occurs before coalescence (Figures S9 and 6a); however, fast coalescence explains most of the steep growth of XRD reflections (Figure S8). Therefore, crystallization temperature should be taken as the onset temperature of the intensity growth regime. This applies to DCS results (extrapolation of peak temperatures for the lower-temperature exotherms, Figure $4 \mathrm{~b}$ ) and to the analysis of XRD width (Scherrer formula, Figure S8).

We take this approach to quantify the size dependence of the crystallization temperature. Figures $6 b-d$ shows XRD intensity-temperature maps for different sizes of GeTe nanoparticles, from which we extract intensity profiles of the main (202) Bragg reflection (Figure 6e). Taking the onset of each intensity profile, we find that the crystallization temperature increases with decreasing the GeTe nanoparticles size (Figure 6f). While such a trend agrees well with the literature on colloidal GeTe nanoparticles (Figure S10), ${ }^{12,13}$ previous reports did not explain this trend.

Here, we fit the size-dependent crystallization of GeTe nanoparticles with two models. The first model is based on the entropy change during the phase transition. ${ }^{29}$ For a melting process, the entropy of fusion, $\Delta S_{\text {melt }}$, can be linked with size- 


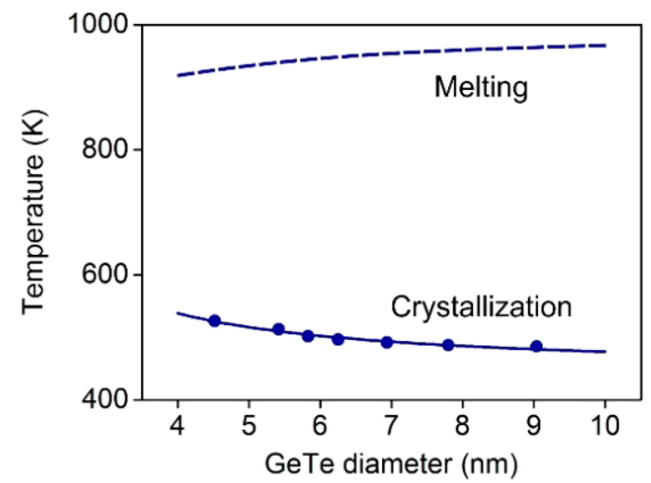

c

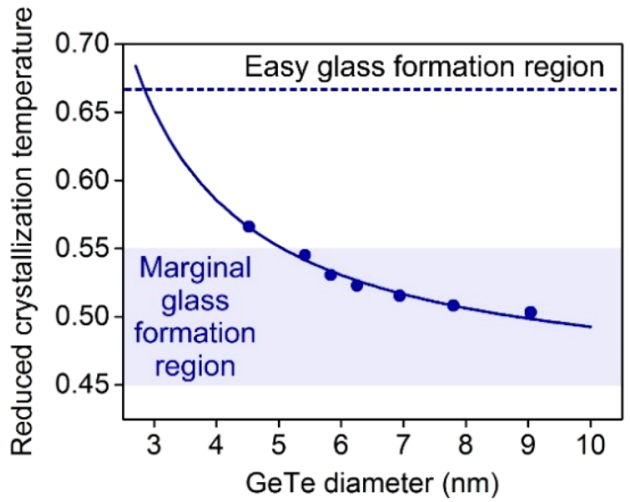

b

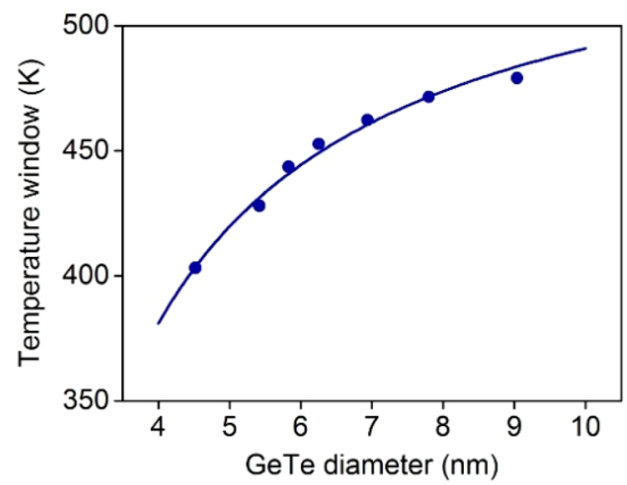

d

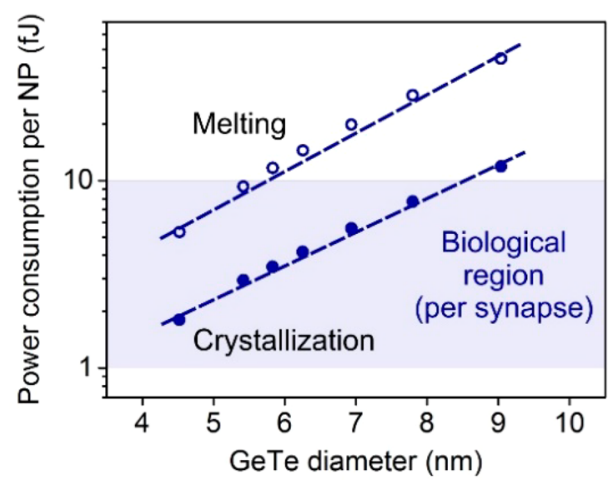

Figure 7. Size effects on (a) crystallization and melting temperatures, (b) temperature window, (c) reduced crystallization temperature (i.e., temperature ratio), and (d) power consumption upon phase transitions for GeTe nanoparticles (NPs) in the sub-10 nm size regime.

dependent melting temperature, $T_{\text {melt }}^{\mathrm{NP}}$ and physical dimensions of material (i.e., diameter of nanoparticles, $d_{\mathrm{NP}}$ ) using the expression:

$$
\frac{T_{\mathrm{melt}}^{\mathrm{NP}}}{T_{\mathrm{melt}}^{\text {bulk }}}=\exp \left(-\frac{2\left(\Delta S_{\mathrm{melt}}-R\right)}{3 R\left(\frac{d_{\mathrm{NP}}}{d_{0}}-1\right)}\right)
$$

where $T_{\text {melt }}^{\text {bulk }}$ is melting point of bulk material, and $d_{0}$ is a critical diameter of nanoparticle (defined as the size of nanoparticle, for which all atoms are surface atoms). ${ }^{29}$ In analogy, we write the size dependent crystallization temperature, $T_{\text {cryst }}^{\mathrm{NP}}$ :

$$
\frac{T_{\text {cryst }}^{\mathrm{NP}}}{T_{\text {cryst }}^{\text {bulk }}}=\exp \left(-\frac{2\left(\Delta S_{\text {cryst }}-R\right)}{3 R\left(\frac{d_{\mathrm{NP}}}{d_{0}}-1\right)}\right)
$$

where we have replaced $\Delta S_{\text {melt }}$ with entropy of crystallization, $\Delta S_{\text {cryst }}$ Taking the bulk crystallization temperature of GeTe from literature $\left(T_{\text {cryst }}^{\text {bulk }}=443.15 \mathrm{~K}\right),{ }^{7}$ we arrive at a simple model with two fitting parameters: entropy of crystallization, $\Delta S_{\text {cryst }}$ and critical diameter, $d_{0}$. We fit this model to the sizedependent crystallization of $\mathrm{GeTe}$ nanoparticles (Figure 6f) and determine important parameters for basic understanding of GeTe crystallization. Namely, we find the entropy of crystallization for $\operatorname{GeTe}\left(\Delta S_{\text {cryst }}=-14.5 \mathrm{~J} /(\mathrm{mol} \cdot \mathrm{K})\right)$ that makes sense: (i) it is negative because ordering increases upon crystallization and (ii) its absolute value is smaller than entropy of fusion $\left(\Delta S_{\text {melt }}=18.0 \mathrm{~J} /(\mathrm{mol} \cdot \mathrm{K})\right){ }^{30}$ which is expected ${ }^{1}$ because of gradual decrease of the disorder parameter for material in its supercooled (i.e., amorphous) state (Figure S11). Furthermore, the extracted critical diameter $\left(d_{0}=0.384\right.$ $\mathrm{nm}$ ) is consistent with the value expected for GeTe. The volume of the sphere with a critical diameter of $0.384 \mathrm{~nm}$ is just slightly smaller than the volume of the smallest GeTe cluster, having at least one nonsurface atom (Figure S12).

We can also explain our data with a second model employing classical nucleation theory, which defines nucleation rate, $J_{\text {cryst }}$ as the number of nuclei per unit volume. ${ }^{31}$ To crystallize a nanoparticle, we need at least one nucleus per nanoparticle. Therefore, $J_{\text {cryst }}$ can be expressed as follows:

$$
J_{\text {cryst }}=\frac{1}{V_{\mathrm{NP}}}=\frac{6}{\pi d_{\mathrm{NP}}^{3}}
$$

where $V_{\mathrm{NP}}$ and $d_{\mathrm{NP}}$ are volume and diameter of spherical GeTe nanoparticle. Alternatively, $J_{\text {cryst }}$ is related to crystallization temperature, $T_{\text {cryst }}$, via the Arrhenius-type equation: ${ }^{31}$

$$
J_{\text {cryst }}=J_{0} \exp \left(-\frac{\Delta G_{\text {cryst }}^{*}}{k_{\mathrm{B}} T_{\text {cryst }}}\right)
$$

Combining 3 and 4, we can relate crystallization temperature and diameter of GeTe nanoparticles via equation, which has two fitting parameters: prefactor, $J_{0}$, and activation energy of nucleation, $\Delta G_{\text {cryst. }}^{*}$ This model displays the observed trend for size-dependent crystallization of GeTe nanoparticles (Figure S11). Smaller sizes of nanoparticles require a higher specific density of nuclei and thus exhibit higher crystallization temperatures. More elaborate nucleation theory models can be built on this basis to account for heterogeneous nucleation, large nucleation rates (i.e., > 1 nucleus per particle), and the effects of surface coverage, etc.

Presented models describe a trend for ultrasmall GeTe nanoparticles (Figure S10), ${ }^{12}$ while slightly higher crystallization temperature is observed for larger GeTe sizes by 
Arachchige et al. and Chen et al. ${ }^{13,32}$ The latter discrepancy can be explained by the nonstoichiometric effects-when the composition of GeTe deviates from Ge:Te 1:1 ratio, the crystallization temperature increases. ${ }^{7}$

Nanodimensional Effects for Phase Change Properties. The size-dependence of the phase transitions in GeTe nanoparticles smaller than $10 \mathrm{~nm}$ highlights the opportunities to design memory devices at the nanoscale. Figure 7 summarizes the effect of size on phase-change properties of GeTe nanoparticles. We calculated melting point depression for GeTe nanoparticles using eq $1^{29}$ and the critical diameter $d_{0}=0.384 \mathrm{~nm}$ extracted from the size-dependent crystallization fitting. While crystallization temperature increases with smaller nanoparticle size, their melting point decreases to lower temperatures (Figure 7a). This increase of the crystallization temperature should lead to improved data retention at room temperature, while the decrease in melting temperature lowers a power consumption of the memory cell. ${ }^{2}$ At the same time, the temperature window (i.e., a difference between $T_{\text {melt }}$ and $T_{\text {cryst }}$ ) remains wider than $400 \mathrm{~K}$ (Figure $7 \mathrm{~b}$ ), ensuring the reliability of memory cell arrays. The size-dependence of these phase transitions may make it possible for materials such as selenides and quaternary telluride compositions that cannot be used in thin film phase change memories due to too low bulk crystallization or too high bulk melting temperature to be used in nanoparticle form phase-change memory devices. ${ }^{3,34}$

The reduced crystallization temperature (i.e., a ratio between $T_{\text {cryst }}$ and $T_{\text {melt }}$ ), which indicates the ease of glass formation, ${ }^{35}$ increases as the size of GeTe nanoparticles decreases (Figure 7c). A size-dependence of reduced crystallization temperature indicates that (i) smaller GeTe nanoparticles are better glass formers (i.e., they can form glass structure at moderate or slow cooling rates); ${ }^{1}$ and (ii) crystallization kinetics of GeTe nanoparticle may attain a size dependence. ${ }^{35}$ Importantly, GeTe nanoparticles larger than 5 $\mathrm{nm}$ can be classified as marginal glass formers, ${ }^{1}$ combining fast crystallization kinetics and stability of supercooled state.

Finally, decreasing the size of phase-change materials leads to improved power efficiency of the device. ${ }^{36,37}$ We calculate the power consumption of a hypothetical memory cell, in which a single GeTe nanoparticle represents a switching phasechange volume (Figure S13). As expected, a power efficiency of such a particle-per-bit device improves as the GeTe size decreases. Even after taking into account the $99 \%$ power losses due to heat dissipation, ${ }^{36}$ the power consumption to crystallize or melt GeTe nanoparticle is on par with energy spent per synaptic event in living organisms (Figure $7 \mathrm{~d}$ ). ${ }^{38}$ Such a comparison is provided to emphasize striking opportunities for colloidal phase-change nanoparticles in ultralow power and high-density phase-change memory devices.

\section{CONCLUSIONS}

This work assessed the potential of colloidal nanoparticles for phase-change memory technology. Using GeTe nanoparticles as a case study, we demonstrated reliable synthesis of colloidal phase change materials and quantified the size-dependence of the crystallization phase transition for the first time. The amide-promoted liquid-phase synthesis enabled accurate size control and large quantity production of GeTe nanoparticles, and it can be extended to other binary and ternary phase change materials. By taking a multipronged approached, we successfully isolated coalescence and crystallization of nanoparticles to quantify the temperature dependence of the crystallization phase transition. We showed that increase in crystallization temperature with decreasing nanoparticle size can be explained using the thermodynamic model or classical nucleation theory. The size-dependent crystallization and melting temperatures in the sub-10 nm size regime offer improved data retention, ultralow power consumption, and fast kinetics of crystallization (i.e., fast write times), highlighting the prospects of nanoparticle phase change materials for memory technology.

\section{ASSOCIATED CONTENT}

\section{Supporting Information}

The Supporting Information is available free of charge on the ACS Publications website at DOI: 10.1021/acs.chemmater.8b02702.

Size and size distribution of GeTe nanoparticles, Figure S1; size distribution and representative TEM images of GeTe nanoparticles, Figure S2; size distribution and representative TEM images of GeTe nanoparticles, Figure S3; typical energy dispersive X-ray spectrum of GeTe nanoparticles, Figure S4; size distributions of GeTe nanoparticles, Figure S5; intensity of (202) Bragg reflection of $\mathrm{GeTe}$ rhombohedral structure while heating GeTe nanoparticles with different ramps, Figure S6; Xray patterns of $\mathrm{GeTe}$ nanoparticles, heated to different temperatures, Figure S7; crystallization temperature estimation for $6 \mathrm{~nm}$ GeTe nanoparticles, Figure S8; TEM analysis of $6 \mathrm{~nm}$ GeTe nanoparticles, Figure S9; size-dependent crystallization of colloidal GeTe nanoparticles, Figure S10; comparison of two fitting models for the size dependence of GeTe crystallization temperature, Figure S11; octahedral $\mathrm{TeGe}_{6}$ cluster, Figure S12; and schematics of particle-per-bit device, Figure S13 (PDF)

\section{AUTHOR INFORMATION}

\section{Corresponding Authors}

*E-mail: vwood@ethz.ch (V.W.).

*E-mail: yaremam@ethz.ch (M.Y.).

ORCID

Maksym Yarema: 0000-0002-2006-2466

\section{Author Contributions}

The manuscript was written through contributions of all authors.

\section{Funding}

This work is financially supported by the Swiss National Science foundation via an Ambizione Fellowship (No. 161249), a research grant (No. 175889), and Quantum Science and Technology NCCR.

\section{Notes}

The authors declare no competing financial interest.

\section{ACKNOWLEDGMENTS}

The authors thank to Mario Mücklich, Weyde Lin, Annina Moser, and Maximilian Jansen for technical assistance. TEM and EDX measurements were performed at the Scientific Center for Optical and Electron Microscopy (ScopeM) of the Swiss Federal Institute of Technology. 


\section{REFERENCES}

(1) Wuttig, M.; Yamada, N. Phase-Change Materials for Rewriteable Data Storage. Nat. Mater. 2007, 6, 824-832.

(2) Burr, G. W.; Brightsky, M. J.; Sebastian, A.; Cheng, H. Y.; Wu, J. Y.; Kim, S.; Sosa, N. E.; Papandreou, N.; Lung, H. L.; Pozidis, H.; Eleftheriou, E.; Lam, C. H. Recent Progress in Phase-Change Memory Technology. IEEE J. Emerg. Sel. Topics Circuits Syst. 2016, 6, 146-162. (3) Raoux, S.; Burr, G. W.; Breitwisch, M. J.; Rettner, C. T.; Chen, Y. C.; Shelby, R. M.; Salinga, M.; Krebs, D.; Chen, S. H.; Lung, H. L.; Lam, C. H. Phase-Change Random Access Memory: A Scalable Technology. IBM J. Res. Dev. 2008, 52, 465-479.

(4) Tolbert, S. H.; Alivisatos, A. P. Size Dependence of a First Order Solid-Solid Phase Transition: The Wurtzite to Rock Salt Transformation in CdSe Nanocrystals. Science 1994, 265, 373-376.

(5) Yarema, M.; Wörle, M.; Rossell, M. D.; Erni, R.; Caputo, R.; Protesescu, L.; Kravchyk, K. V.; Dirin, D. N.; Lienau, K.; von Rohr, F.; Schilling, A.; Nachtegaal, M.; Kovalenko, M. V. Monodisperse Colloidal Gallium Nanoparticles: Synthesis, Low Temperature Crystallization, Surface Plasmon Resonance and Li-Ion Storage. J. Am. Chem. Soc. 2014, 136, 12422-12430.

(6) Reiss, P.; Carrière, M.; Lincheneau, C.; Vaure, L.; Tamang, S. Synthesis of Semiconductor Nanocrystals, Focusing on Nontoxic and Earth-Abundant Materials. Chem. Rev. 2016, 116, 10731-10819.

(7) Okabe, T.; Nakagawa, M. Crystallization Behavior and Local Order of Amorphous $\mathrm{Ge}_{\mathrm{x}} \mathrm{Te}_{1-\mathrm{x}}$ Films. J. Non-Cryst. Solids 1986, 88, 182-195.

(8) Perniola, L.; Sousa, V.; Fantini, A.; Arbaoui, E.; Bastard, A.; Armand, M.; Fargeix, A.; Jahan, C.; Nodin, J. F.; Persico, A.; Blachier, D.; Toffoli, A.; Loubriat, S.; Gourvest, E.; Beneventi, G. B.; Feldis, H.; Maitrejean, S.; Lhostis, S.; Roule, A.; Cueto, O.; Reimbold, G.; Poupinet, L.; Billon, T.; De Salvo, B.; Bensahel, D.; Mazoyer, P.; Annunziata, R.; Zuliani, P.; Boulanger, F. Electrical Behavior of PhaseChange Memory Cells Based on GeTe. IEEE Electron Device Lett. 2010, 31, 488-490.

(9) Chen, Y.; Wang, G.; Song, L.; Shen, X.; Wang, J.; Huo, J.; Wang, R.; Xu, T.; Dai, S.; Nie, Q. Unraveling the Crystallization Kinetics of Supercooled Liquid GeTe by Ultrafast Calorimetry. Cryst. Growth Des. 2017, 17, 3687-3693.

(10) Boschker, J. E.; Wang, R.; Calarco, R. GeTe: A Simple Compound Blessed with a Plethora of Properties. CrystEngComm 2017, 19, 5324-5335.

(11) Samanta, M.; Biswas, K. Low Thermal Conductivity and High Thermoelectric Performance in $(\mathrm{GeTe})_{1-2 \mathrm{x}}(\mathrm{GeSe})_{\mathrm{x}}(\mathrm{GeS})_{\mathrm{x}}$ : Competition between Solid Solution and Phase Separation. J. Am. Chem. Soc. 2017, 139, 9382-9391.

(12) Caldwell, M. A.; Raoux, S.; Wang, R. Y.; Philip Wong, H.-S.; Milliron, D. J. Synthesis and Size-Dependent Crystallization of Colloidal Germanium Telluride Nanoparticles. J. Mater. Chem. 2010, 20, 1285-1291.

(13) Arachchige, I. U.; Soriano, R.; Malliakas, C. D.; Ivanov, S. A.; Kanatzidis, M. G. Amorphous and Crystalline GeTe Nanocrystals. Adv. Funct. Mater. 2011, 21, 2737-2743.

(14) Polking, M. J.; Zheng, H.; Ramesh, R.; Alivisatos, A. P. Controlled Synthesis and Size-Dependent Polarization Domain Structure of Colloidal Germanium Telluride Nanocrystals. J. Am. Chem. Soc. 2011, 133, 2044-2047.

(15) Schulz, S.; Heimann, S.; Kaiser, K.; Prymak, O.; Assenmacher, W.; Brüggemann, J. T.; Mallick, B.; Mudring, A.-V. Solution-Based Synthesis of GeTe Octahedra at Low Temperature. Inorg. Chem. 2013, 52, 14326-14333.

(16) Buck, M. R.; Biacchi, A. J.; Popczun, E. J.; Schaak, R. E. Polymer-Assisted Synthesis of Colloidal Germanium Telluride NanoOctahedra, Nanospheres, and Nanosheets. Chem. Mater. 2013, 25, 2163-2171.

(17) Alivisatos, A. P. Perspectives on the Physical Chemistry of Semiconductor Nanocrystals. J. Phys. Chem. 1996, 100, 1322613239 .
(18) Dick, K.; Dhanasekaran, T.; Zhang, Z.; Meisel, D. SizeDependent Melting of Silica-Encapsulated Gold Nanoparticles. J. Am. Chem. Soc. 2002, 124, 2312-2317.

(19) Yarema, M.; Yarema, O.; Lin, W. M. M.; Volk, S.; Yazdani, N.; Bozyigit, D.; Wood, V. Upscaling Colloidal Nanocrystal Hot-Injection Syntheses via Reactor Underpressure. Chem. Mater. 2017, 29, 796803.

(20) Lin, Q.; Yun, H. J.; Liu, W.; Song, H.-J.; Makarov, N. S.; Isaienko, O.; Nakotte, T.; Chen, G.; Luo, H.; Klimov, V. I.; Pietryga, J. M. Phase-Transfer Ligand Exchange of Lead Chalcogenide Quantum Dots for Direct Deposition of Thick, Highly Conductive Films. J. Am. Chem. Soc. 2017, 139, 6644-6653.

(21) Nag, A.; Kovalenko, M. V.; Lee, J.-S.; Liu, W.; Spokoyny, B.; Talapin, D. V. Metal-free Inorganic Ligands for Colloidal Nanocrystals: $\mathrm{S}^{2-}, \mathrm{HS}^{-}, \mathrm{Se}^{2-}, \mathrm{HSe}^{-}, \mathrm{Te}^{2-}, \mathrm{HTe}^{-}, \mathrm{TeS}_{3}{ }^{2-}, \mathrm{OH}^{-}$, and $\mathrm{NH}^{2-}$ as Surface Ligands. J. Am. Chem. Soc. 2011, 133, 10612-10620.

(22) Yarema, M.; Pichler, S.; Sytnyk, M.; Seyrkammer, R.; Lechner, R. T.; Fritz-Popovski, G.; Jarzab, D.; Szendrei, K.; Resel, R.; Korovyanko, O.; Loi, M. A.; Paris, O.; Hesser, G.; Heiss, W. Infrared Emitting and Photoconducting Colloidal Silver Chalcogenide Nanocrystal Quantum Dots from a Silylamide-Promoted Synthesis. ACS Nano 2011, 5, 3758-3765.

(23) Yarema, O.; Bozyigit, D.; Rousseau, I.; Nowack, L.; Yarema, M.; Heiss, W.; Wood, V. Highly Luminescent, Size- and Shape-Tunable Copper Indium Selenide Based Colloidal Nanocrystals. Chem. Mater. 2013, 25, 3753-3757.

(24) Yarema, O.; Yarema, M.; Bozyigit, D.; Lin, W. M. M.; Wood, V. Independent Composition and Size Control for Highly Luminescent Indium-Rich Silver Indium Selenide Nanocrystals. ACS Nano 2015, 9, 11134-11142.

(25) Yarema, O.; Yarema, M.; Lin, W. M. M.; Wood, V. Cu-In-Te and Ag-In-Te Colloidal Nanocrystals with Tunable Composition and Size. Chem. Commun. 2016, 52, 10878-10881.

(26) Saldanha, P. L.; Lesnyak, V.; Manna, L. Large Scale Syntheses of Colloidal Nanomaterials. Nano Today 2017, 12, 46-63.

(27) Clark, T. J.; Reed, J. S. Kinetic Processes Involved in the Sintering and Crystallization of Glass Powders. J. Am. Ceram. Soc. 1986, 69, 837-846.

(28) Chen, B.; ten Brink, G. H.; Palasantzas, G.; Kooi, B. J. SizeDependent and Tunable Crystallization of GeSbTe Phase-Change Nanoparticles. Sci. Rep. 2016, 6, 39546.

(29) Zhang, Z.; Zhao, M.; Jiang, Q. Melting Temperatures of Semiconductor Nanocrystals in the Mesoscopic Size Range. Semicond. Sci. Technol. 2001, 16, L33-L35.

(30) Sosso, G. C.; Miceli, G.; Caravati, S.; Giberti, F.; Behler, J.; Bernasconi, M. Fast Crystallization of the Phase Change Compound GeTe by Large-Scale Molecular Dynamics Simulations. J. Phys. Chem. Lett. 2013, 4, 4241-4246.

(31) Liu, J.; Xu, X.; Brush, L.; Anantram, M. P. A Multi-Scale Analysis of the Crystallization of Amorphous Germanium Telluride Using Ab Initio Simulations and Classical Crystallization Theory. J. Appl. Phys. 2014, 115, 023513.

(32) Chen, B.; de Wal, D.; ten Brink, G. H.; Palasantzas, G.; Kooi, B. J. Resolving Crystallization Kinetics of GeTe Phase-Change Nanoparticles by Ultrafast Calorimetry. Cryst. Growth Des. 2018, 18, 10411046.

(33) Wang, K.; Steimer, C.; Detemple, R.; Wamwangi, D.; Wuttig, M. Assessment of Se Based Phase Change Alloy as a Candidate for Non-Volatile Electronic Memory Applications. Appl. Phys. A: Mater. Sci. Process. 2005, 81, 1601-1605.

(34) Choi, M.; Choi, H.; Kim, S.; Ahn, J.; Tae Kim, Y. Lattice Distortion in $\mathrm{In}_{3} \mathrm{SbTe}_{2}$ Phase Change Material with Substitutional Bi. Sci. Rep. 2015, 5, 12867.

(35) Turnbull, D. Under What Conditions Can a Glass be Formed? Contemp. Phys. 1969, 10, 473-488.

(36) Fong, S. W.; Neumann, C. M.; Wong, H.-S. P. Phase-Change Memory - Towards a Storage-Class Memory. IEEE Trans. Electron Devices 2017, 64, 4374-4385. 
(37) Lee, S.-H.; Jung, Y.; Agarwal, R. Highly Scalable Non-Volatile and Ultra-Low-Power Phase-Change Nanowire Memory. Nat. Nanotechnol. 2007, 2, 626-630.

(38) Xu, W.; Min, S.-Y.; Hwang, H.; Lee, T.-W. Organic CoreSheath Nanowire Artificial Synapses with Femtojoule Energy Consumption. Sci. Adv. 2016, 2, e1501326. 